\title{
Can high SARS-CoV-2 adult vaccination rates help protect unvaccinated children? Evidence from a unique rapid mass vaccination campaign
}

jörg paetzold ( $\sim$ joerg.paetzold@sbg.ac.at)

University of Salzburg

\section{Research Article}

Keywords: SARS-CoV-2 transmission, local mass vaccination campaign, herd immunity, protection of unvaccinated individuals

Posted Date: October 7th, 2021

DOl: https://doi.org/10.21203/rs.3.rs-958479/v1

License: (c) (1) This work is licensed under a Creative Commons Attribution 4.0 International License.

Read Full License 
1 Can high SARS-CoV-2 adult vaccination rates help protect unvaccinated children?

2 Evidence from a unique rapid mass vaccination campaign

3

4 Hannes Winner ${ }^{1 *}$, Janine Kimpel $^{2 \#}$, Florian Krammer $^{3}$, Dorothee von Laer ${ }^{2}$, Jörg Paetzold ${ }^{1 * \#}$ 5

6 Affiliation

$7 \quad{ }^{1}$ University of Salzburg, Department of Economics, Residenzplatz 9, A-5010 Salzburg, Austria

$8{ }^{2}$ Institute of Virology, Department of Hygiene, Microbiology and Public Health, Medical 9 University of Innsbruck, Peter-Mayr-Str. 4b, 6020 Innsbruck, Austria

$10{ }^{4}$ Department of Microbiology, Icahn School of Medicine at Mount Sinai, One Gustave L. Levy 11 Place, Box 1124 New York, NY 10029

$13 *$ contributed equally

14

15 \# corresponding author:

16 Jörg Paetzold University of Salzburg, Department of Economics, Residenzplatz 9, A-5010 17 Salzburg, Austria

18 Email: Joerg.Paetzold@sbg.ac.at

19

20 Janine Kimpel Institute of Virology, Department of Hygiene, Microbiology and Public Health, 21 Medical University of Innsbruck, Peter-Mayr-Str. 4b, 6020 Innsbruck, Austria

22 Email: Janine.Kimpel@i-med.ac.at 
Background: Mass vaccination has the potential to bring the COVID-19 pandemic to a halt by not only protecting individuals who have been vaccinated but also by providing cross-protection to unvaccinated individuals, such as children. However, this indirect protection effect from a vaccinated population onto an unvaccinated group is extremely difficult to observe in realworld situations.

Methods: We studied cross-protection to unvaccinated individuals following an unprecedented rapid mass vaccination campaign in Europe. After a large outbreak of B.1.351 (Beta) in the district of Schwaz in Austria, the government offered every adult (16+) citizen of the district a vaccination with BNT162b2 between the $11^{\text {th }}$ and $16^{\text {th }}$ of March 2021. After this week, around $70 \%$ of the adult population of Schwaz had received their first dose, which made Schwaz the first widely inoculated region in Europe. The cohort of children under the age of 16 remained entirely unvaccinated (EMA only approved the vaccine for 12-15 year-olds on the $28^{\text {th }}$ of May). This local mass vaccination campaign created a situation in which the vaccination coverage of the adult population sharply differed at the district border of Schwaz, while the coverage of those below the age of 16 remained the exact same. We compared SARS-CoV-2 cases among the adult population as well as children in Schwaz with case numbers of the same age cohorts from control regions. First, we compared Schwaz with a control group of other Austrian districts highly similar to Schwaz in many socio-demographic characteristics as well as in infection spread prior to the mass vaccination campaign. Second, we compared local populations residing along the border of Schwaz which live in the very same geographic area but with different vaccination coverage because they were not included in the vaccination campaign.

Interpretation: Prior the mass vaccination campaign, we observed very similar infection spread across all age cohorts in Schwaz and the control regions. Around 3-4 weeks after the campaign, infections started to diverge between Schwaz and the control regions. While the difference was largest among the population aged 16-50 years (which was offered vaccination in the campaign), we also observed a statistically significant reduction in cases among the group of unvaccinated children. Our findings are robust to changes in the control group, as well as controls of a rich set of time and region specific effects.

\section{Policy implications:}

Our results constitute one of the first evidence of an indirect cross-protection effect from a group of vaccinated individuals to an unvaccinated group (in our case children). Given that in many countries the proposition to keep schools open during the academic year 2021/22 is a top priority, this evidence of community-protection is highly policy relevant.

\section{Introduction}

Mass vaccination campaigns against SARS-CoV-2 hold the promise to bring the pandemic to a halt by achieving high rates of vaccination coverage. However, in almost all countries vaccines against SARS-CoV-2 are still not available for very young age cohorts, for instance those below the age of 12. In addition, some parents are hesitant regarding potential risks and benefits of inoculating their kids, which suggests that vaccination coverage among the very young might remain modest even after the approval of a vaccine for small children. This raises the important question about the herd immunity threshold where vaccinated individuals provide cross-protection to unvaccinated individuals in the community $(1,2)$. If this indirect vaccination effect exists, a high coverage among older cohorts may protect younger cohorts from infection (3). Given that many countries are currently trying to find ways to ensure that schools remain 
open during the new 2021/22 school year, studying this community-protection effect is also 71 highly policy relevant.

To answer this question, we exploited a unique rapid mass vaccination campaign. Following a breakout of B.1.351 in the district of Schwaz (Austria), the government of Austria and BioNTech joined forces and supplied 100,000 extra vaccine doses of BNT162b2 to rapidly mass vaccinate the entire adult population (16+) of Schwaz (4,5). After the first campaign weekend in mid March 2021, around 70\% of the adult population of Schwaz had received their first dose of BNT162b2, which made Schwaz the first widely inoculated region in Europe. In contrast, the rest of the country had a very low vaccination coverage (first dose) of around 10\% at that time (4). This local mass vaccination campaign created a situation in which the vaccination coverage of the adult population sharply differed at the district border of Schwaz, while the coverage of those below the age of 16 remained the exact same, basically zero (EMA approved the first vaccine for those under 16 only on May $28^{\text {th }}$ 2021). In our analysis we compared Schwaz with (i) a control group of highly similar districts, and (ii) with populations residing in municipalities along the border of Schwaz which were just excluded from the campaign to study the effect of cross-protection to unvaccinated children under the age of 16 .

So far, evidence of this indirect protection effect from mass vaccination against SARS-CoV-2 is extremely scarce. Only one study estimated this indirect effect of population-wide mass vaccination coverage (6), focusing on the community-level temporal variation in vaccine coverage in Israel and relating this figure to the temporal variation of positive SARS-CoV-2 tests. The underlying variation in vaccine coverage between the two time periods the study employed was modest, ranging from a 5 to a maximum 20 percentage point change in the fraction of vaccinated individuals. In contrast, the variation in vaccination coverage we analyzed is considerably larger, with coverage jumping from around $10 \%$ to more than $70 \%$ within one weekend, compared to the control regions. Thus, our analysis is very well suited to study the potential effect of community-level protection. In addition, our setting very accurately reflects the situation many countries are facing right now, hitting the ceiling of vaccination readiness at around $60-70 \%$ of vaccination coverage.

\section{Methods}

Our retrospective observational study used municipality- and district-level age-specific incidence data from the Austrian epidemiological reporting system (Österreichisches Epidemiologisches Meldesystem, EMS). The study has been reviewed by the Ethics Committee of the University of Salzburg stating that no approval was needed as all data has been received and processed in an anonymized fashion. Our analysis is based on a difference-in-difference design in which we compare unvaccinated age-cohorts (below 16) in the district Schwaz with the same age-cohort in the control regions before and after the mass vaccination campaign. We study the period from January 2021 until the $28^{\text {th }}$ of May, the day when EMA approved BNT162b2 also for children older than 12 years. Until this date, all children below the age of 16 in both the treatment and control group were unvaccinated. For comparison, we also examined incidence rates for individuals aged 16-50 years, who are likely to represent the population that interacts the most with the unvaccinated cohort of under 16 years of age (7).

To estimate the indirect effect of the mass vaccination campaign on the cross-protection to unvaccinated teenagers we used two different methods: First, we used the synthetic control method (SC), which is widely applied in causal analysis (8), and also in recent health and COVID-19 research $(9,10)$. The synthetic control group is constructed through a data-driven process in which weights are assigned to all 91 Austrian districts in order to approximate as closely as possible the pre-treatment characteristics of Schwaz. The choice of the weights is based on the SARS-CoV-2 infection spread prior to the vaccination campaign and additional covariates such as population size, geographical area size and the number of municipalities 
within a district (further details are provided in (5)). We compared age-group specific incidence rates between Schwaz and its synthetic counterpart before and after the mass vaccination campaign. This allowed to estimate what would have happened to the group of unvaccinated below 16 year-olds in Schwaz in the absence of the mass vaccination campaign.

Second, we made use of our very fine-grained geographical data to compare Schwaz with adjacent municipalities just outside the border of the district. This ensures that the population living in these border municipalities share many geographical and socio-demographic characteristics (e.g., local mobility) with the population of Schwaz but were excluded from the mass vaccination campaign. We employed an event-study model based on a difference-indifference (DID) design to measure the impact of the campaign on the incidence among the people with age below 16 years from Schwaz relative to the same age-group from the border municipalities (11). Border municipalities were included in the study when a direct road link between the respective border municipality and the district of Schwaz existed. Dependent variable was the number of positive SARS-CoV-2 cases for the age-group below 16 . We estimated a two-way fixed-effects model including an indicator variable for municipalities located in Schwaz as the treated units. We calculated for each week $k$ the DID in the 7-day moving average of new infections (per 100,000 inhabitants) for children aged below 16 years from the bordering municipalities and Schwaz. The regression equation is given by

$$
y_{i t, w}=\delta_{i}+\delta_{w}+\sum_{k=-6}^{-1} \beta_{k} D_{i t, w}+\sum_{k=1}^{11} \beta_{k} D_{i t, w}+\epsilon_{i t, w},
$$

where $y_{i t, w}$ denotes the 7-day moving average of new infections (per 100,000) for children below 16 years from municipality $i$ (Schwaz or border municipalities) and day $t$, which is nested in week $w . \delta_{i}$ and $\delta_{w}$ denote municipality- and week-fixed effects, and $D_{i t, w}$ is the treatment variable taking a value of 1 for municipalities in Schwaz and 0 for border municipalities just outside of Schwaz. $k$ in the sum operators indicate leads (first sum) and lags (second sum) of the treatment effect. $\varepsilon_{i t, w}$ is a classical i.i.d. error term. Standard errors are clustered at the municipality level. Our coefficients of interest are the $\beta_{k}$, which measure the difference in the outcome variable (e.g., daily infections per 100,000 for children below 16 years) between Schwaz and the neighboring border municipalities at a given week $k$ relative to the omitted reference category, which is the week of the first dose of the campaign $\left(11^{\text {th }}\right.$ to $16^{\text {th }}$ of March).

Finally, we calculated the overall average effect of the community-level protection from the vaccination campaign in Schwaz relative to the border municipalities using a standard twoperiod DID analysis. Specifically, we estimated one post-treatment effect that comprised the average reduction in daily infections for children below 16 years over all post-campaign weeks starting 14 days after the roll-out of the first dose (which is approximately the time period after which first effects of BNT162b2 materialized in the original clinical trial (12). Each treatment effect is reported as a percent reduction of new cases due to the vaccination campaign (relative to the border municipalities), along with the corresponding 95\%-CI.

\section{Results}

\section{Impact of the mass vaccination campaign on vaccine coverage}

To illustrate the stark difference in vaccine coverage following the mass vaccination campaign we calculated the shares of individuals aged 16-50 years that received the first and second dose, respectively. We focus on the vaccination rates of individuals between 16-50 years old because this is probably the most relevant population group regarding a potential cross-protection effect on the unvaccinated cohort of children under 16 (7). Figure 1 plots vaccination rates of this age group for the district of Schwaz as well as for all other Tyrolian districts (pooled together). The massive impact of the mass vaccination campaign in Schwaz vis-à-vis the other districts is 
striking. Prior to the first dose of the campaign $\left(11^{\text {th }}\right.$ to $16^{\text {th }}$ of March), vaccination coverage among the 16-50 year-olds was exactly the same between Schwaz and everywhere else, with around 5\%. After the first campaign week, this vaccination coverage increased more than tenfold in Schwaz, to around 60\%. When three weeks later the second dose was administered ( $8^{\text {th }}$ to $11^{\text {th }}$ of April), Schwaz likely became Europe's region with the highest vaccine coverage among this age group. Notice that EMA approved the first vaccine for children aged 12-15 years only on May $28^{\text {th }} 2021$. Thus, during the entire time period of our study there were basically no vaccinations (besides of single cases of off-label usage) among children below 16, neither in the control nor treatment region. Having an identically unprotected children population interacting with an adult population with dramatically different vaccination coverage is what makes our study so unique.

\section{Schwaz vs. synthetic control group}

To examine the indirect effect impact of this stark difference in vaccination coverage we used the daily number of SARS-CoV-2 infections at the district level as the respective outcome variable. We calculated the cumulative daily infections from the second week of January 2021 onwards. We employed the synthetic control group method which allowed us to estimate what would have happened to Schwaz in the absence of the mass vaccination campaign (further details are provided in (5)).

184 Figure 2 depicts the difference in cumulative daily infections by age group between the synthetic control group and Schwaz. First, it shows that for both age groups the treatment and the (synthetic) control group had very similar spread of SARS-CoV-2 infections prior to the mass vaccination campaign, confirming that treatment- and control-groups are highly comparable in both age groups. Second, around 3-4 weeks after the first dose, the sum of infections started to diverge in both age groups. At the end of the observational period $\left(28^{\text {th }}\right.$ of May), we observed a difference of around 990 cumulative daily infections per 100,000 inhabitants for the adults aged 16-50 years (i.e., 2,750 cumulative daily infections per 100,000 in the control group, and 1,760 in the Schwaz). This figure translates into a difference of around $56.1 \%$. For children below 16 years, we found a difference of 634 avoided infections $(2,220$ in the control group versus 1,586 in Schwaz), giving a relative difference of around $40.0 \%$. Since this age group was not part of the vaccination campaign in Schwaz, it is not surprising that the difference between treatment- and control group is larger for adults than for children below 16 years. However, we still observed a large difference between the synthetic control group and Schwaz regarding children below the age of 16, suggesting a population-level effect in the sense that vaccinated individuals provide cross-protection to unvaccinated individuals in the community. In sum, Figure 2 indicates a systematic and substantial indirect protection effect of vaccinating a majority of the relevant adult population.

\section{Schwaz vs. bordering municipalities}

In addition to the analysis of Austrian districts based on the synthetic control group, we also compared the district of Schwaz with adjacent municipalities located along the district border. Thus, this analysis examined infections among local populations residing within the same geographic area, but with stark differences in vaccine coverage after the campaign.

Figure 3 plots the weekly treatment effects of an event-study model according to equation (1), capturing the difference between Schwaz and the border municipalities relative to the reference period (week of the first dose of the campaign, $11^{\text {th }}$ to $16^{\text {th }}$ of March). Figure 3a is based on infections of adults aged 16-50 years, and Figure 3b on infections of children below 16 years. Both panels of the figure show that in the weeks prior to the mass vaccination campaign, the differences between Schwaz and the border municipalities were not statistically different from zero. Very similar to our evidence based on synthetic control districts, we found that the number 
of new cases in Schwaz for both age groups decreased significantly relative to the border municipalities after the second dose. Calculating the DID effect over the entire observational period, we found a larger treatment effect for adults than for children, being around $-75.1 \%$ (95\%-CI: $-85.8--47.8 \%$ ). For children below 16 years, we observed a treatment effect of about $-64.5 \%$ (95\%-CI: $-82.0--30.2 \%)$, indicating again a systematic and substantial indirect protection effect of vaccination.

\section{Discussion}

This retrospective observational study examined the impact of community-level protection on incidence rates of the group of unvaccinated children. We exploited a unique rapid mass vaccination campaign to estimate this indirect protection effect on unvaccinated children under the age of 16. As a control group we used the same age-cohort from comparable but untreated districts and border municipalities (i.e., with no community-level protection) which followed very similar trends in infections spread prior to the campaign.

We first documented a massive vaccine uptake that raised coverage from around $5 \%$ to $60 \%$ of the population between 16 and 50 years old after the campaign. Our analysis revealed that the massive rollout of BNT162b2 mRNA vaccine in Schwaz was associated with a significant reduction in new SARS-CoV-2 infections among the age-cohort of unvaccinated children of around $40-65 \%$ relative to the same age-cohort from the control regions.

Our study has potential limitations. First, our study is not a randomized clinical trial but an observational study, which may be influenced by confounders such as lockdown policies. While almost all non-pharmaceutical interventions (such as school measures, or curfew restrictions) were identical for Schwaz and the control regions, there was an additional SARS-CoV-2 test requirement between the 11th of March and the 8th of April when crossing the border of the district. This test requirement may have affected the spread of infections. However, we analyzed for every district of Austria which had the same test requirement (in total five other districts) if infection numbers dropped in a similar magnitude as they did in Schwaz, but none of them experienced a decline in any comparable way after the test requirement (5). Second, while our difference-in-difference design controls for time-varying general trends over time in infection spread (such as a third wave), we cannot directly account for changing individual behavior such as vaccinated individuals being less mindful of social-distancing measures. However, an analysis of mobility data does not show large differences between Schwaz and the control districts (5). In fact, even if the vaccinated adult population of Schwaz may have indeed obeyed less to social-distancing rules after the campaign, we still observed a significant indirect effect on the unvaccinated group of children. This observed reduction of cases of an unvaccinated group despite a potentially less mindful vaccinated population is policy-relevant since it reflects a scenario many countries are likely to experience once a large share of their adult population is vaccinated.

To sum up, our results constitute one of the first evidence of an indirect (cross-)protection effect from a rapid COVID-19 mass vaccination campaign on an unvaccinated group. Given that the vaccination coverage in Schwaz was very similar to the level of vaccination coverage many countries are facing right now (around 70\%), the results of our study can also applied to other regions and countries.

\section{References}

1. Kadkhoda K. Herd immunity to COVID-19. American Journal of Clinical Pathology 2021; 155, 471-472. 
2. Fontanet A, et al. COVID-19 herd immunity: where are we. Nature Reviews Immunology 2020; 20, 583-584.

3. Harvey RA, et al. Association of SARS-CoV-2 seropositive antibody test with risk of future infection. JAMA Internal Medicine 2021; 181, 672-679.

4. AGES, Österreichische Agentur für Gesundheit und Ernährungssicherheit GmbH. SARSCoV-2-Varianten in Österreich. https://www.ages.at/themen/krankheitserreger/coronavirus/sars-cov-2-varianten-inoesterreich/ (accessed 26.04.2021).

5. Paetzold JM, Kimpel J, Bates K, Hummer M, Krammer F, von Laer D, Winner H. The effects of rapid mass vaccination against SARS-CoV-2 and its Variants-of-Concern: Evidence from an early VoCs hotspot. Research Square, doi: 10.21203/rs.3.rs-741944/v1

6. Milman O., et al. Community-level evidence for SARS-CoV-2 vaccine protection of unvaccinated individuals. Nature Medicine 2021; 27, 1367-1369.

7. Béraud G, et al. The French connection: the first large population-based contact survey in France relevant for the spread of infectious diseases. PLoS ONE 2021; 10 (e0133203).

8. Cunningham, S. Causal Inference, New Haven \& London 2021

9. Kreif N, Grieve R, Hangartner D, Turner AJ, Nikolova S, et al. Examination of the Synthetic Control Method for Evaluating Health Policies with Multiple Treated Units. Health Economics 2016; 25 (12): 1514-1528.

10. Cho SW. Quantifying the impact of nonpharmaceutical interventions during the COVID19 outbreak: The case of Sweden. The Econometrics Journal. 2020 Sep;23(3): 323-44

11. Dobkin C, Finkelstein A, Kluender R, Notowidigdo MJ. The economic consequences of hospital submissions. American Economic Review 2018; 108 (2): 308-352.

12. Polack FP, Thomas SJ, Kitchin N, Absalon J, Gurtman A, et al. Safety and Efficacy of the BNT162b2 mRNA Covid-19 Vaccine. N Engl J Med 2020; 383 (27): 2603-2615.

\section{Data availability}

For this study we used data from the Austrian epidemiological reporting system (Österreichisches Epidemiologisches Meldesystem, EMS). These data are collected by the Austrian National Public Health Institute (Gesundheit Österreich GmbH, GÖG), and is provided to the researchers through a restricted-access agreement. Future access to this dataset can be considered through direct application for data access to the GÖG. Standard epidemiological analyses were conducted using standard commands in STATA/SE 16.1 (ref. 36).

\section{Acknowledgments}

We are grateful to Daniela Schmid and Lukas Richter from AGES for providing SARS-CoV-2 qPCR datan. We also would like to thank Elmar Rizzoli and Thomas Geiler from "Amt der Tiroler Landesregierung" for providing sequencing and vaccination data for the state of Tyrol. Finally, we thank the GÖG for data assistance.

\section{Author contributions}

J.P. codesigned the study, performed statistical analyses and cowrote the first draft of the article. H.W. conceived and codesigned the study, performed the statistical analyses and cowrote the first draft of the article. J.K., F.K. and D.V.L. codesigned the study. J.P. and H.W. equally 
303 contributed to data collection and acquisition, as well as database development. All authors 304 contributed to the discussion and interpretation of the results, and to the writing of the 305 manuscript. All authors have read and approved the final manuscript.

\section{Competing interest}

307 The Icahn School of Medicine at Mount Sinai has filed patent applications relating to SARS308 CoV-2 serological assays and NDV-based SARS-CoV-2 vaccines which list Florian Krammer 309 as co-inventor. Mount Sinai has spun out a company, Kantaro, to market serological tests for 310 SARS-CoV-2. Florian Krammer has consulted for Merck and Pfizer (before 2020), and is 311 currently consulting for Pfizer, Seqirus and Avimex. The Krammer laboratory is also collaborating with Pfizer on animal models of SARS-CoV-2. For all other authors, no conflicts of interests exist. The funders had no role in the design of the study; in the collection, analyses, or interpretation of data; in the writing of the manuscript, or in the decision to publish the results. 


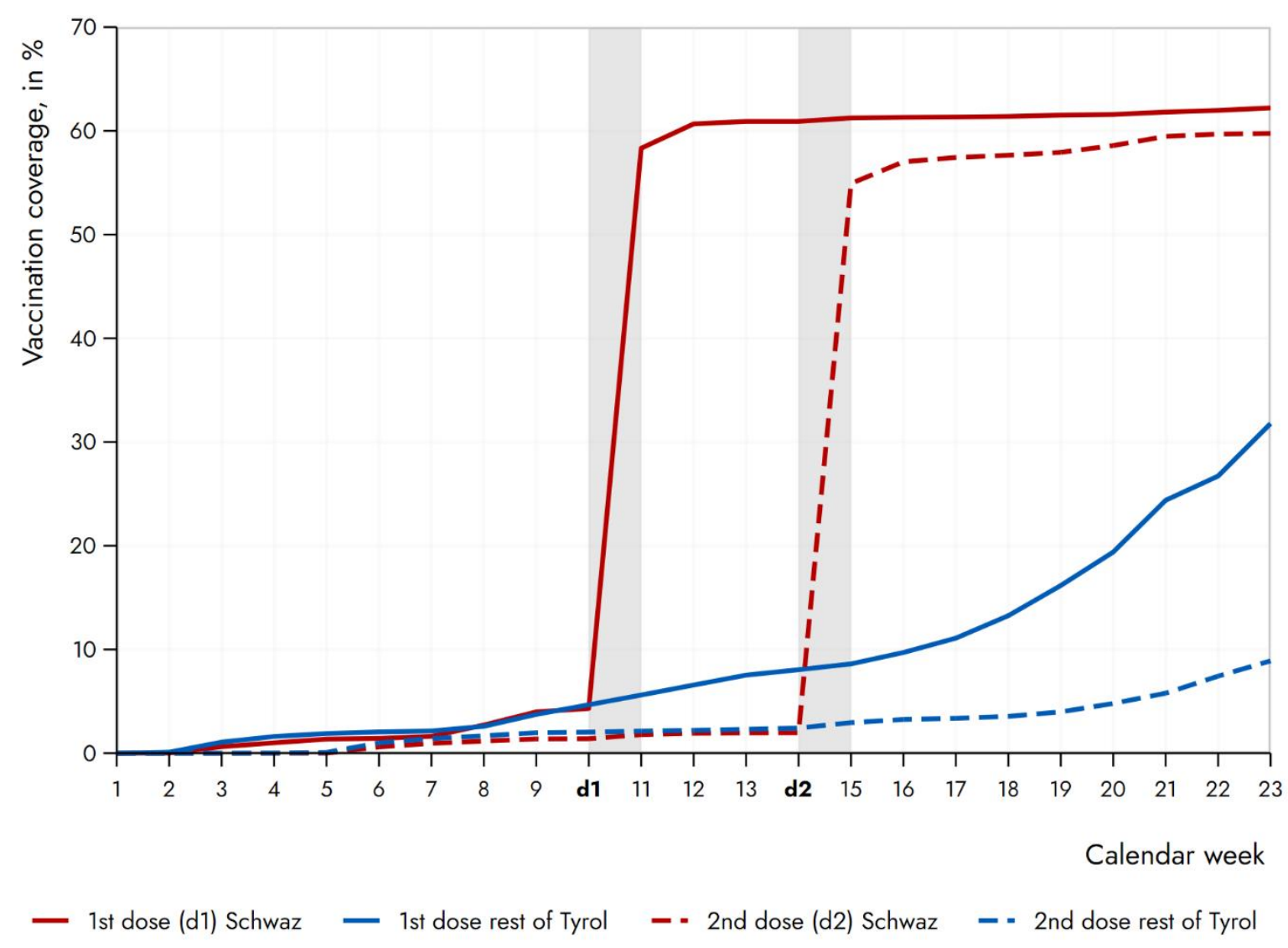

Figure 1. Vaccination coverage of people with age between 16 and 50 years in Schwaz and the rest of 320 Tyrol

321 The figure displays the shares of adults between 16 and 50 years that received the first (solid line) and second dose (dashed line) of vaccination, respectively. Schwaz is plotted in red, while the other (eight) Tyrolian districts are pooled and depicted in blue. The shaded areas indicate the period of the first (d1: $11^{\text {th }}$ to $16^{\text {th }}$ of March 2021, calendar week 10) and the second (d2: $8^{\text {th }}$ to $11^{\text {th }}$ April 2021, calendar week 14) roll-out of mass vaccination. 


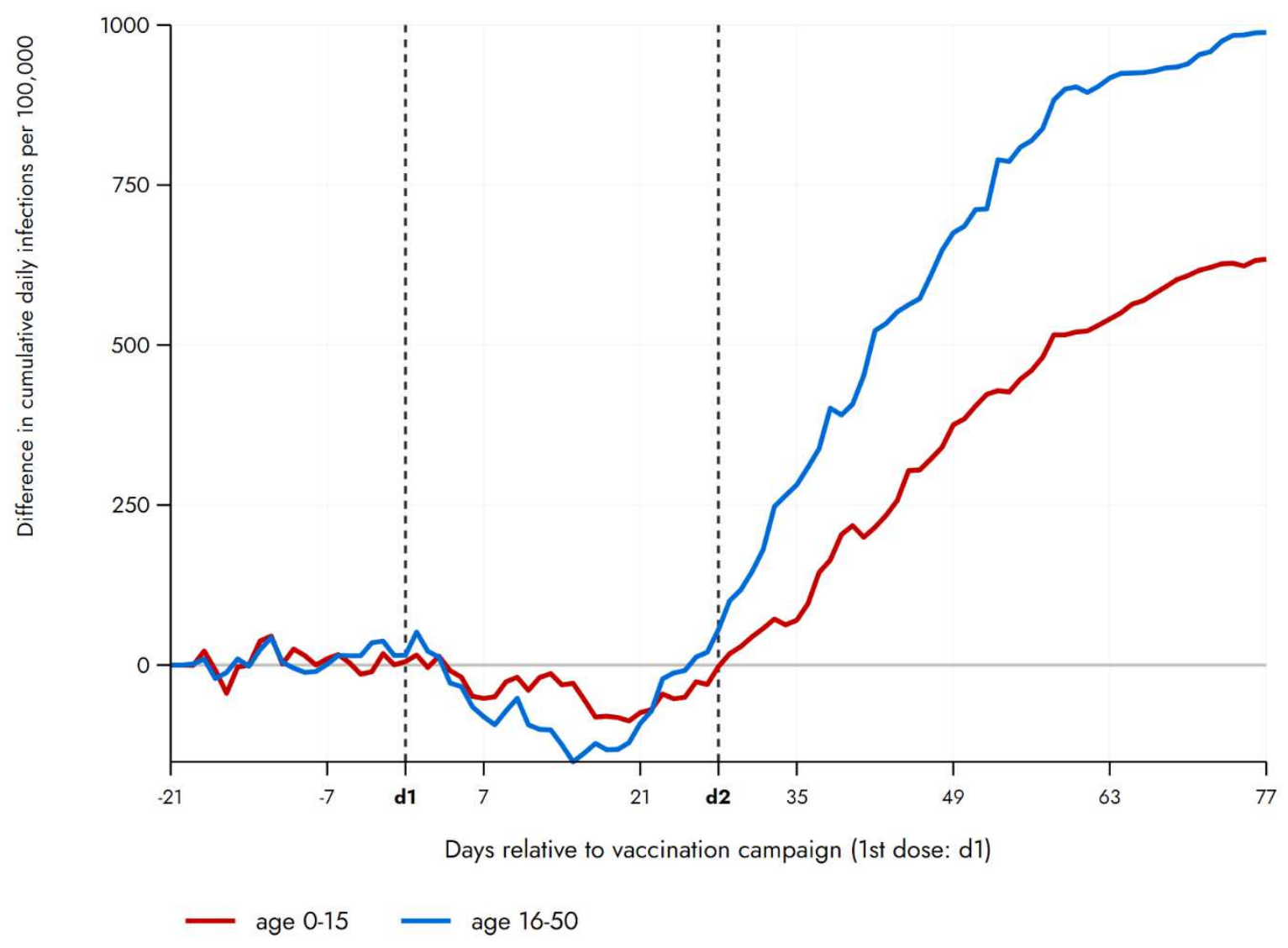

Figure 2. Difference in cumulative daily infections by age group between synthetic control group and Schwaz

330 The figure depicts for the two age groups in the sample the difference in cumulative daily infections (per 100,000) between the synthetic control group and Schwaz. A positive difference indicates higher infection rates for the control group than for Schwaz. The horizontal axis shows the number of days relative to vaccination campaign (dose 1). The pre-treatment period started 21 days before the first dose, the post-treatment period ended 77 days after the first dose (i.e., 28th of May). The vertical dashed lines campaign. 


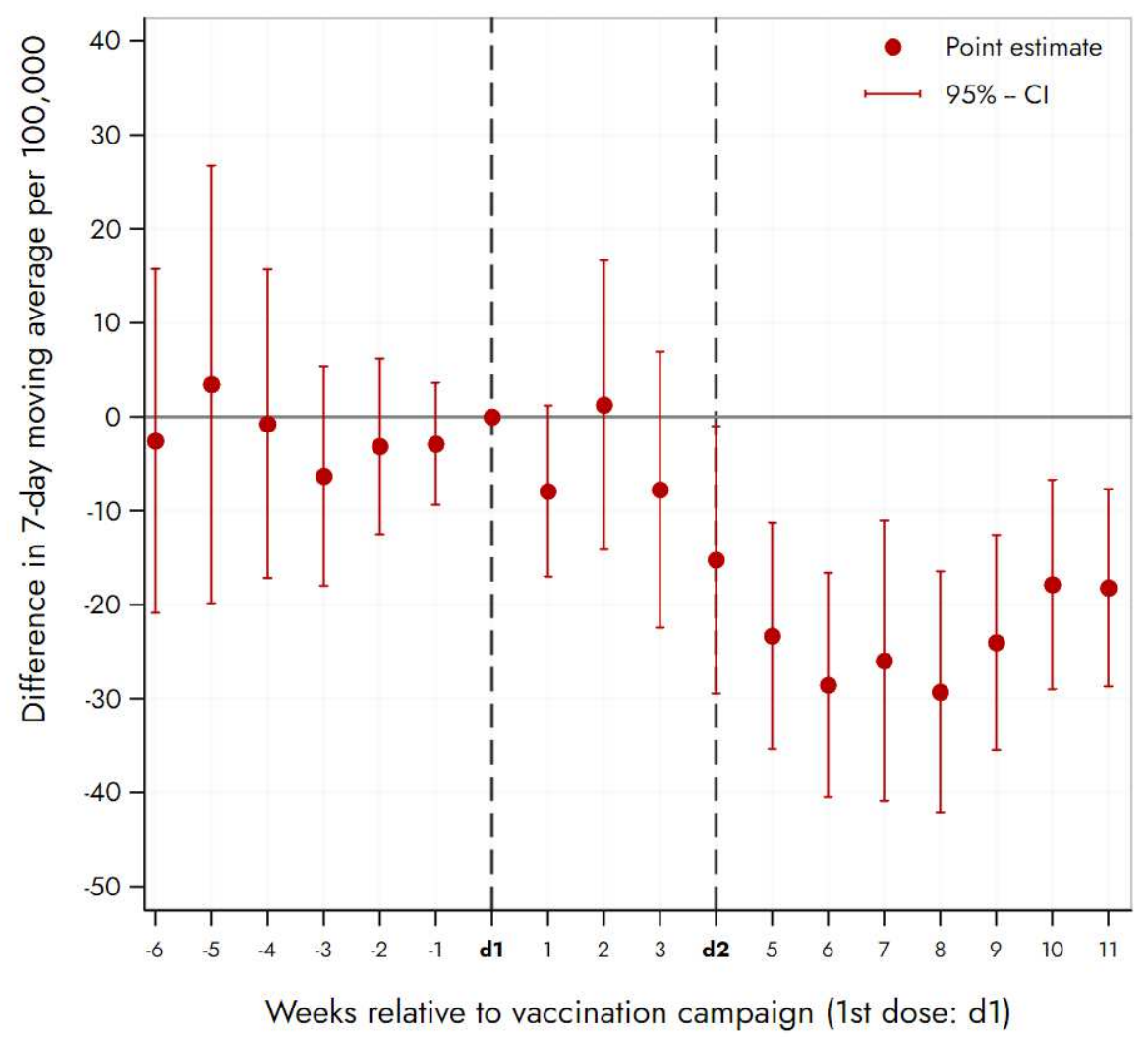

b

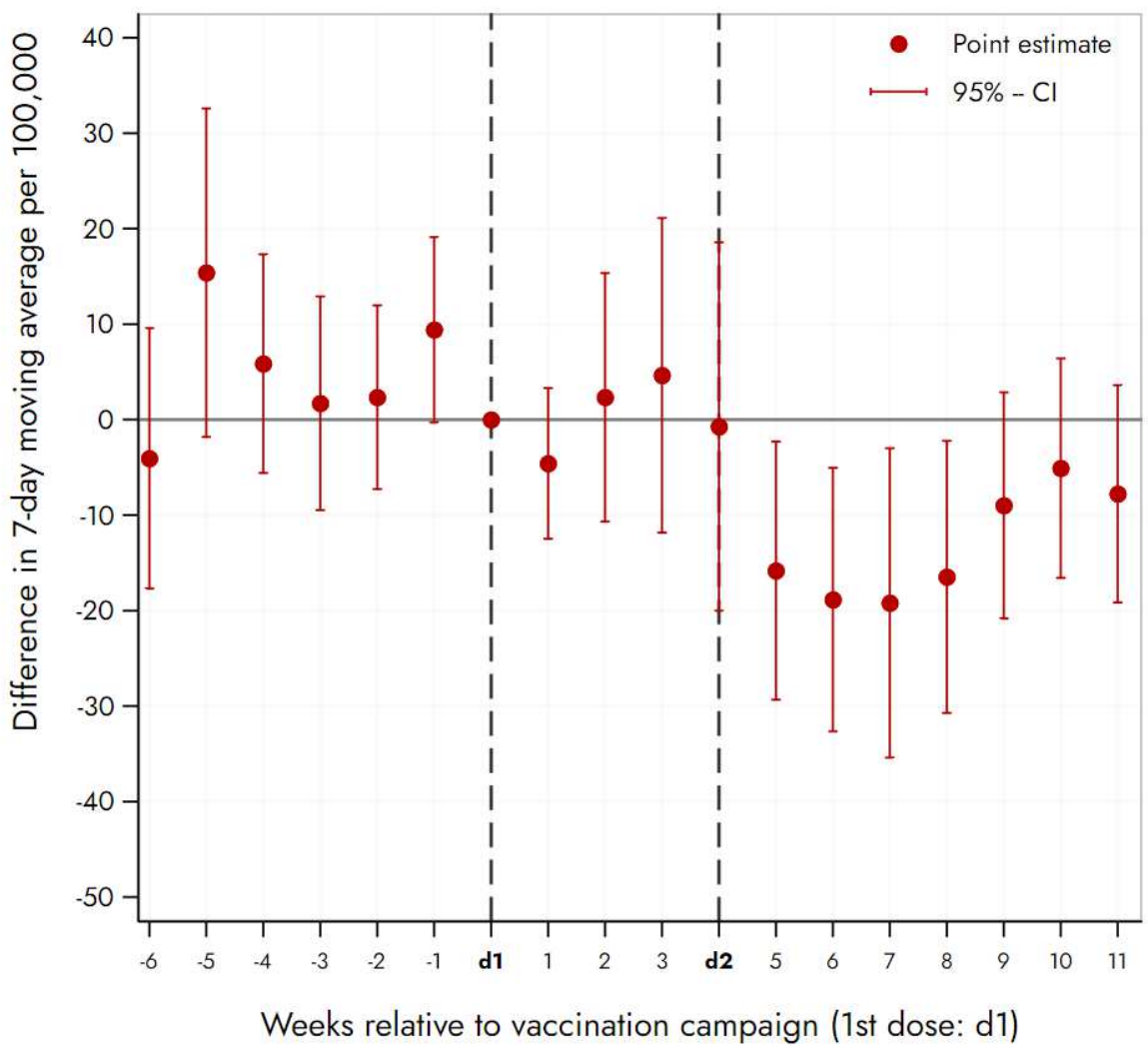

Figure 3. Daily infections with SARS-CoV-2 for (a) people with age between 16 and 50 years, and (b) people with age below 16 years

The figure displays the results from regression equation (1) and uses the 7-day moving average of daily cases (per 100,000) as outcome variable for Schwaz and its bordering municipalities. a refers to people between 16 and 50 years, and $\mathbf{b}$ to people below 16 years. The plotted coefficients represent the weekly difference in the 7-day moving average of new cases between Schwaz and the border municipalities relative to the reference period (week when dose 1 of campaign was administered which is calendar week 10 of 2021). The coefficient for each week is shown together with the 95\%-confidence interval. The horizontal axis displays the number of weeks relative to vaccination campaign (dose 1). The vertical dashed lines represent the first dose (d1) and the second dose (d2) administered as part of the mass vaccination campaign. 